\title{
The Role of Periosteum in Mastoid Surgery
}

Alaa A. Abou-Bieh, MD

Department of Otolaryngology, Faculty of

Medicine, Mansoura University,

Mansoura, Egypt

\section{Introduction}

This work is to investigate the importance of the integrity and pattern of the periosteal lining of the mastoid cavity in the outcome and sequel of the different types of the mastoid surgeries by detecting its condition during the surgery and correlating it to the postoperative prognosis.

\section{Material and Methods}

This was a prospective study included the revision mastoid surgeries operated upon between the years 2010 and 2015 in two tertiary referral hospitals and a private practice set up after was approved by the national review board. It included 122 cases (74 were right side, 46 were left side, 101 were males, 21 were females and 8 were pe- diatric cases). Cholesteatoma cases including the second look cases were 27 cases and chronic suppurative otitis media (CSOM) cases of non cholesteatoma type were 95 cases. Different surgical techniques were used as needed for the cases situations and included 54 canal wall-up mastoidectomies (including 4 second look operations) with or without tympanoplasty and 68 canal wall-down mastoidectomies. Surgeries were done by different surgeons but the author was involved in most of them. Intra-operative multiple curettages from the unhealthy-looking bone in direct relation to pathologic findings as granulations, polypi, and cholesteatoma were collected and histopathologically studied to determine the nature of the bony tissue and the status of the periosteum. The 
procedures associated to the creation of the histopathological were followed the description of Paparella et al.11 Preoperative computed tomography (CT) scans for both revision and primary surgery for every case were evaluated whenever possible to correlate the radiological findings to the clinical and histopathological findings.

\section{Results}

Periosteal changes or deficiency usually associated with surrounding bony pathological changes could be detected in all cases. Periosteal layer deficiency or absence in one or more areas of the mastoid cavity was present in almost all cases, and it was always replaced by granulation tissue or unhealthy bony tissue. Periostitis was suspected in 23 cases $(18 \%)$. It was appreciated by the chronic changes in the periosteal layer with localized areas of new bone formation. The new bone formation in the subperiosteal layer denoting that a lesion (usually a subperiosteal abscess) raises the periosteum away from the bone with new ossification on the original bone that is growing at a rate which is faster than the periosteum can grow or expand, so instead of dimpling, the periosteum tears away and provides ossification, very much like the concept of Codman triangle. In some cases, some areas of the periosteal reaction was sever enough to resemble the aggressive rapid bone formation not the slow benign one with new bone lamellated or amorphous, single layered or multilayered, solid or spiculated, these sever changes usually associated with sever recurrent primary disease, granuloma or aggressive cholesteatoma. This picture of non-suppurative ossifying periostitis with proliferation of periosteum and subperiosteal bone formation can be compared to the picture of the periostitis ossificans or sclerosing osteomyelitis of Garré but on a limited scale. However, the areas of aggressive periosteal reaction with inflammation of the soft-tissue around it can be compared to the florid reactive periostitis ossificans. The surrounding bone showed histo- pathological picture of osteitis and osteomyelitis of different levels of severities in 69 cases (57\%). The histological studies showed some spaces filled with inflammatory exudate which does not progressed to actual formation of pus, inflammatory cells as neutrophils, polymorphonuclear leukocytes, also lymphocytes and plasma cells, degenerated osteoblasts bordering the bony trabeculae, areas of bone resorption and some sequestrum and involucrum like formation denoting chronic osteomyelitis or chronic focal sclerosing osteomyelitis (condensing osteitis) with proliferation rather than destruction due to the dense of the trabeculae of the bone bordered by an active layer of osteoblasts with areas of focal osteoblastic activity and the mosaic pattern which is due to repeated periods of resorption followed by repair. The soft tissue surrounding is fibrous and shows proliferating fibroblast, small capillaries, lymphocytes, plasma cells, and polymorphonuclear leukocytes. These also could be appreciated on studying some of the cases CT scans on a retrograde bases.

These findings could be related mainly to the extent and type of mastoid pneumatization, type, stage and activity of the primary, residual and / or recurrent disease, patient age and technique of first surgery. Studying whatever available CT scans for the case could appreciate these concepts. When the mastoid was of sclerotic or diploic cellularity type before the primary surgery, the pathological changes was very sever. This was also the in pediatric cases, extensive cholesteatoma and extensive primary surgery. The least pathological changes were when the primary surgery was performed in cellular mastoid especially in adults. When mastoid cavity was obliterated by any of the periosteal flaps during primary surgery, the pathological changes were very minimal.

The average follow-up of cases after the revision surgery was twelve months. The prognosis of cases was very relative to the periosteum and bone 
pathological status with directly proportional, the worst the periosteum and bone situation was encountered during surgery the worst the prognosis of the case with surgery failure, granulation tissue or polypi formation and / or wet unhealthy mastoid cavity.

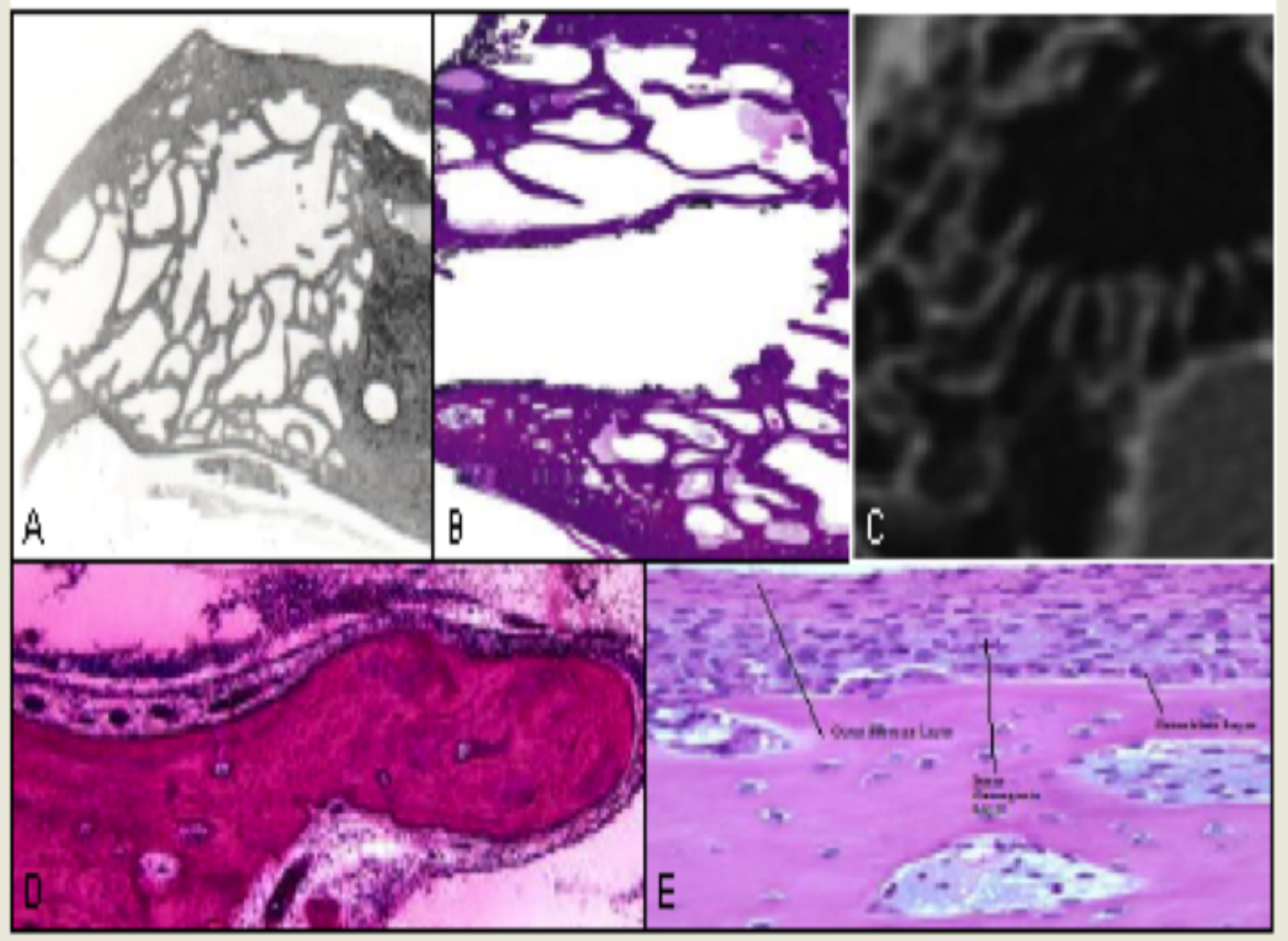

Figure 1 Normal Mastoid. A) Cancellaus bane with trabeculae farming mataid air celt. B) Celloidin preparation, HaE stained. C) CT san axial. D) Bone trabealae with normal periosteum high m占nification 20X. E) N armal periasteum high m疋nification 30X. 


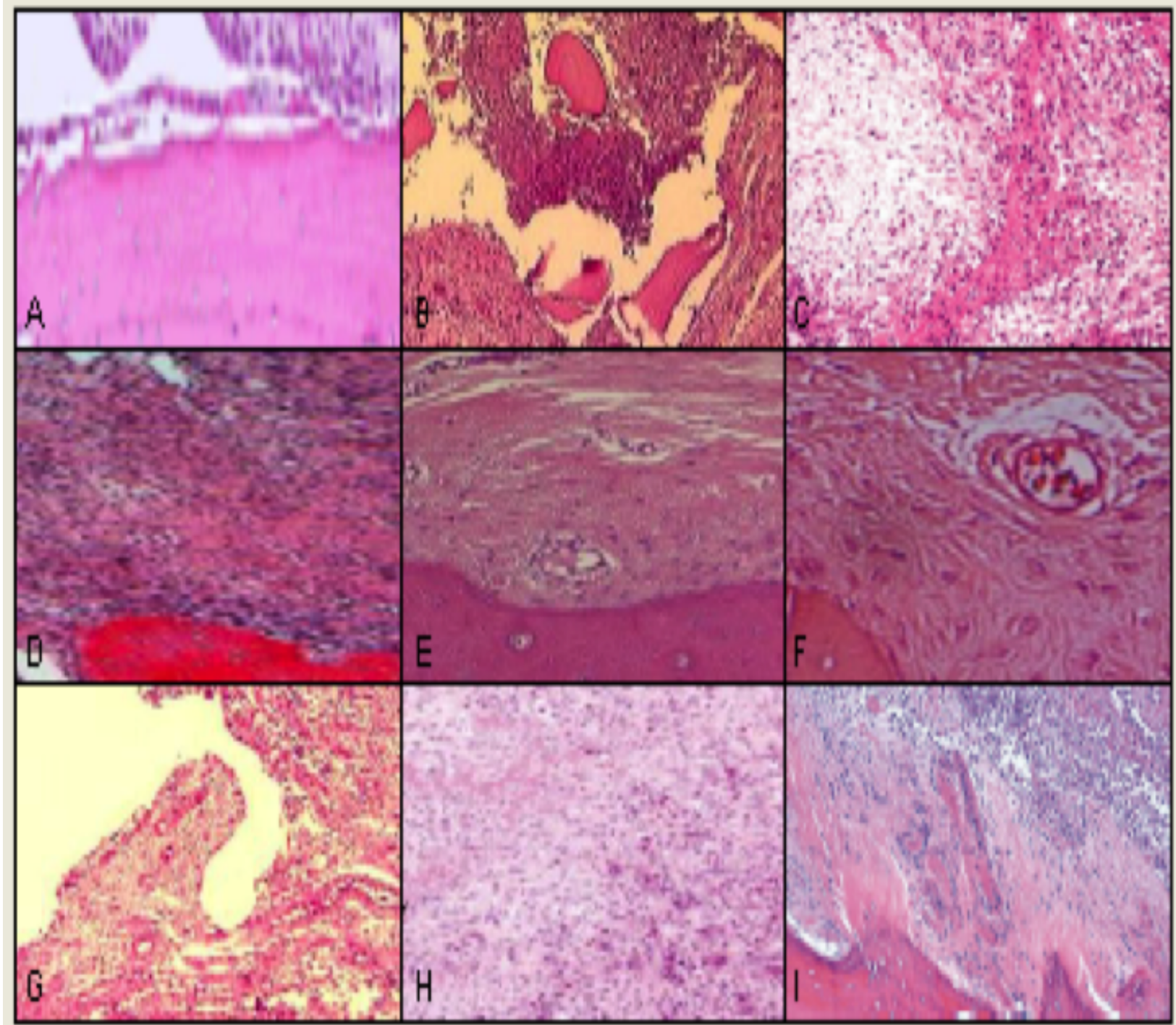

Figure 2. Periosteal and Bone Patholog\%. A) Perioteal deficiency. Bi) Periotea deficiency with bone necrosis C) Periostijs. D) Prol liferdive periostitis E) Proliferd.ive periostits new bone formation. F) Proliferative periostitis new bone formation high magrificdion 20X. Gị New boneformzion. H) Oteitis. I) osteonyy ditis. 


\section{Discussion}

The primary objective in the surgical management of chronic suppurative otitis media (CSOM) with or without cholesteatoma has been usually the eradication of disease, as well as ensuring a dry and safe ear. ${ }^{1}$ Together with avoiding complications and preserving or restoring hearing as much as possible, they forming the main measurable outcomes of mastoid surgery. However eradication of the disease and creating dry, safe ear is the basic factor for considering surgery either successful or not. ${ }^{2}$ The high rate of the surgical failures including the residual and recurrent disease have led to the hundreds of the published research, suggesting methods and protocols of treatment and have built controversies in the surgical management include the choice in surgical approach, that is, canal wall down (CWD) or canal wall up (CWU), and the need for staged operations. ${ }^{3,4}$ Many factors have been proposed and discussed as causes of failure to create dry and safe ear, among them is a periosteal lining or coverage of the mastoid cavity and mastoid air cells system. 5,6 However, the role of periosteal lining was mainly discussed and investigated regarding the mastoid cavity obliteration by neoosteogenesis under the periosteal flap as histological investigations relating fracture-healing process have shown that intra-membranous bone formation begins under the periosteum in a few days after fracture formation and bridging between fracture fragments begins in 4 weeks. ${ }^{7}$ Calcification foci were observed indicating osteoblastic activity in histological investigations of a cavity wall biopsy taken from a patient in 6 months after surgery using one type of the periosteal flaps and new bone formation filling the mastoidectomy cavity under the flap was detected in the postoperative temporal bone CT images taken in the 1st, 12th and 24th months. ${ }^{5}$

The presence of healthy and integral periosteal lining of the mastoid cavity either the primary lining or a secondary lining via periosteal type flap has many effects and benefits in mastoid surgeries and healing beside enhancing the neoosteogenesis. The mastoid bone is formed of a communicated bony air-cells system of varies degrees and distribution. 8 Histologically, it is a cancellous bone with trabeculae forming small cavities: the mastoid cells, lined by respiratory mucosa forming a mucoperiosteal layer, with a fatty layer and hematopoietic tissues and permeated by blood vessels. ${ }^{9,10}$ Compared to the paranasal sinuses, the mastoid air-cells system is very similar anatomically and histologically and subsequently the same prognostic factors can be applied on it intra- and postoperatively. It is very well established now that the restoring ventilation and drainage without total removal of the mucosa from the major sinuses together with the status and integrity of the periosteal lining of the sinuses are the major factors in the success of functional endoscopic sinus surgery. ${ }^{12-14}$ This based on the concept that, if the periosteum could be preserved on the surface of the bone, normal mucosa could regenerate over the periosteum without formation of granulation or scar tissue and without reducing the size of the cavity, changing it from radical to functional surgery. ${ }^{12}$ The other factor which makes the periosteum an important element in mastoid surgery is the pathological nature of the chronic suppurative otitis (CSOM) media as a mucoperiosteal disease which makes the periosteal affection and involvement inevitable. ${ }^{15}$ In addition, the nature and extent of the temporal bone and mastoid pneumatization which is usually limited or hypocellular in case of chronic otitis media (COM) would force the surgery and drilling to take place in a sclerotic bone which should result in an artificial cavity not lined by periosteum and should heal by granulation tissue and normal mucosa will never grow in it. The hypocellular or sclerotic mastoid would result from a number of middle ear diseases which are associated with pathologic 
bone modeling, either formative or resorptive as experimental studies on middle ear infection have shown varying degrees of both osteoresorption and osteoneogenesis and this is conceivably in support of the environmental theory of mastoid pneumatization, claiming inflammatory disease as the cause of a sclerotic mastoid. ${ }^{16-17}$

conclusions:

Healthy integral periosteal lining of the mastoid cavity seems to be an essential factor for the cavity stability and surgery outcome. Detailed understanding of the mastoid cavity development, anatomy, and histology together with the studying of the preoperative CT scans helps in preserving the integrity of the cavity periosteum during surgery. Periosteal flaps of different types obliterating the mastoid cavity can be of great value when the pathology involved the periosteum, it is impossible to find or leave intact periosteum or it is inevitable to breach the periosteum.

\section{References}

1. Sheehy JL, Brackmann DE. Surgery of chronic otitis media. In: English GM, ed. Otolaryngology. Philadelphia: Lippincott Williams and Wilkins; 1994: 20.

2. Wilson KF, Hoggan R N, Shelton C. Tympanoplasty with intact canal wall mastoidectomy for cholesteatoma: longterm surgical outcomes. Otolaryngol Head Neck Surg 2013; 149(2):292-5.

3. Syms MJ, Luxford WM. Management of cholesteatoma: status of the canal wall. Laryngoscope 2003; 113(3):443-8.

4. Kuo CY, Huang BR, Chen $\mathrm{HC}$, et al. Surgical results of retrograde mastoidectomy with primary reconstruction of the ear canal and mastoid cavity. Biomed Res Int 2015; 2015: 517035.
5. Ucar C. Canal wall reconstruction and mastoid obliteration with composite multifractured osteoperiosteal flap. European Archives of Oto-Rhino-Laryngology 2006; 263 (12):1082-6.

6. Kahramanyol M. Fascioperiosteal flap and neoosteogenesis in radical mastoidectomy. Ear Nose Throat J 1992;71(2):70-2, 75-7.

7. Einhorn TA. The cell and molecular biology of fracture healing. Clin Orthop Relat Res 1998 Oct;(355 Suppl):S7-21.

8. Allam AF. Pneumatization of the temporal bone. Ann Otol Rhinol Laryngol 1969 Feb;78 (1):49-64.

9. Merchant SN, Nadol Jr JB. Schuknecht's Pathology of the Ear, 3rd edition. USA PMPH; 2010.

10. Soares HB, Lavinsky L. Histology of sheep temporal bone. Braz J Otorhinolaryngol 2011 Jun;77(3):285-92.

11. MM, Lamey SF, Goycoolea MV. Histology and pathology of the ear. In: Paparella MM, Shumrick A. Otolaryngology. 3rd ed. Philadelphia: WB Saunders; 1991. p.419-38.

12. Kikawada $\mathrm{T}$, Nonoda $\mathrm{T}$, Matsumoto $\mathrm{M}$, et al. Treatment of intractable diseased tissue in the maxillary sinus after endoscopic sinus surgery with high-pressure water jet and preservation of the periosteum. Arch Otolaryngol Head Neck Surg 2000 Jan;126(1):55-61.

13. Kennedy DW. Prognostic factors, outcomes and staging in ethmoid sinus surgery. Laryngoscope. 1992 Dec; 102(12 Pt 2 Suppl 57):1-18.

14. May M, Levine HL, Schaitkin B, et al. Result 
of surgery. In Levine HL ed. Endoscopic Sinus Surgery. New York, NY Thieme-Stratton Inc 1993;176- 192.

15. Meyerhoff W. Pathology of chronic suppurative otitis media. Ann Otol Rhinol Laryngol Head and Neck Surg 1988; 97(Suppl. 130):21-4.
16. Cayé-Thomasen $P$, Hermansson $A$, Tos $M$, et al. Bone modeling dynamics in acute otitis media. Laryngoscope 1999 May; 109(5):723-9.

17. Flohr S, Kierdorf U, Schultz M.Differential diagnosis of mastoid hypocellularity in human skeletal remains. Am J Phys Anthropol 2009 Nov;140(3):442-53. 